\title{
An analytical solution based on mobility and multicriteria optimization for access selection in heterogeneous environment
}

\author{
C AMALI $^{1, *}$, DHANASREE JAYAPRAKASH ${ }^{2}$ and B RAMACHANDRAN ${ }^{2}$ \\ ${ }^{1}$ Valliammai Engineering College, Chennai 603203, India \\ ${ }^{2}$ SRM University, Chennai 603203, India \\ e-mail: amali.vec@gmail.com; dhanasreejayaprakash@gmail.com; ramachandran.b@ktr.srmuniv.ac.in
}

MS received 25 August 2016; revised 3 March 2017; accepted 17 March 2017; published online 14 October 2017

\begin{abstract}
Providing global connectivity with high speed and guaranteed quality at any place and any time is now becoming a reality due to the integration and co-ordination of different radio access technologies. The internetworking of existing networks with diverse characteristics has been considered attractive to meet the incredible development of interactive multimedia services and ever-growing demands of mobile users. Due to the diverse characteristics of heterogeneous networks, several challenges have to be addressed in terms of quality of service (QoS), mobility management and user preferences. To achieve this goal, an optimal network selection algorithm is needed to select the target network for maximizing the end user satisfaction. The existing works do not consider the integration of utility function with mobile terminal mobility characteristics to minimize ping-pong effects in the integrated networks. An integrated multicriteria network selection algorithm based on multiplicative utility function and residual residence time (RRT) estimation is proposed to keep the mobile users always best connected. Multiplicative weighted utility function considers network conditions, application QoS and user preferences to evaluate the available networks. In this paper, the proposed scheme is implemented with two mainstreams (pedestrian users and high-velocity users). For high-velocity users, RRT and adaptive residence time threshold are also considered to keep the probability of handover failures and unnecessary handovers within the limits. Monte-Carlo simulation results demonstrate that the proposed scheme outperforms against existing approaches.
\end{abstract}

Keywords. Utility function; mobility management; residual residence time; adaptive residence time threshold; user-centric network selection.

\section{Introduction}

Next Generation Wireless Networks (NGWN) expects a user to be able to access services independent of his/her location in order to realize the concept of 'always best connected (ABC)' [1]. ABC concept includes many components such as network discovery, network selection, handover execution, mobility management, etc., in which network selection places a milestone in the NGWN to select the best suitable network in order to cope with the growing demands of high-data-rate applications. In traditional network-centric approach, operators hold a firm control over users to achieve profitable resource utilization. However, NGWN requires user-centric approach in which the users will have access to the available resources according to the application quality of service $(\mathrm{Q} o \mathrm{~S})$, user preference and mobility characteristics. This is where the need for user-centric network selection algorithm to

*For correspondence provide vertical handover decision (VHD) in heterogeneous wireless networks becomes important.

The primary role of the mobility management is to render seamless mobility and ubiquitous service access by minimizing service disruption time whenever handover occurs. The vertical handover (VHO) process comprises three phases, namely system discovery, handover decision and handover execution. The main focus of this work is on network selection process, being part of the handover decision module. The VHD process decides when and to which network the handover needs to be performed in a heterogeneous environment when the user is on the move. The decision-making process of handover may be centralized or decentralized (i.e., the handover decision may be made at the network or mobile terminal-MT; [2]. From the decision process view, one can find at least three different kinds of handover decisions. They are 1. networkcontrolled handover (NCHO), 2. mobile-controlled handover (MCHO) and 3. mobile-assisted handover (MAHO). In traditional network selection strategies, only the networks can make VHD based on the MT's measurement 
report. However, $\mathrm{NCHO}$ is not suitable to incorporate the use of multiple interface connections and user preferences in the overlapping region of different networks.

In this paper, mobile-controlled network assisted handover is considered because only the MT has the knowledge about the networks available in the coverage area and user preferences. Among the different approaches addressing the multicriteria decision-making problem provided in the literature, an approach based on utility theory and mobility is considered. If the MT velocity and moving pattern are irregular, more unnecessary handover can occur [3]. When the MT is moving with high velocity, it is necessary to find out how much time the MT will stay in the target network. If the estimated residual residence time (RRT) is less than adaptive residence time threshold (ARTT), handover to such a network is not beneficial as it requires more number of handover to complete the ongoing service. In this work, we determine the adaptive threshold values $T_{1}, T_{2}$ and $T_{3}$ as a function of velocity, residue angle, residence time and designed value of performance metrics in order to keep the probability of handovers, handover failures and unnecessary handovers within the limits.

The rest of this paper is organized as follows. The related works with their motivation and shortcomings are discussed in section 2. The system model of the proposed approach, the sequence of operations and analysis of network selection process are described in section 3. Section 4 presents the numerical results to evaluate the efficiency of the proposed scheme. The conclusion and future extension are presented in section 5 .

\section{Related works}

This section provides an overview of recent research works on network selection algorithm for VHD in heterogeneous wireless networks.

Multicriteria VHD algorithm is presented in Mehbodniya et al [4] to maximize the end user satisfaction in which fuzzy extension of the techniques for order preference by similarity to ideal solution is used to rank the available networks. Mehbodniya et al [5] proposed VHO decision algorithm based on Fuzzy VIsekriterijumsko KOmpromisno Rangiranje, which incorporates the use of parallel fuzzy logic controllers with minimum number of rules to estimate the necessity of handover and also to select the target network. Determining the most suitable weights for the criteria is the main problem in multiattribute decisionmaking algorithms. The fuzzy control theory is used to evaluate the performance of available networks by considering the metrics RSS, bandwidth, network delay and user preference in He et al [6]. Singhrova and Prakash [7] developed a neuro-fuzzy-based vertical handover decision (VHD) algorithm, which considers received signal strength, velocity, bandwidth, number of users, battery level and coverage. An adaptive neuro-fuzzy inference system improves the throughput and QoS by reducing ping-pong effects compared with the fuzzy technique.

Kaleem et al [8] presented the implementation of fuzzy pre-processing module in which the handover necessity is performed to trigger the handover process in the proper time while achieving the end user's satisfaction by providing uninterrupted QoS to the users. A new intelligent VHO algorithm is presented in Amali et al [9] that uses fuzzy logic to estimate the necessity of handover and also to determine a new point of attachment in order to fulfil the end user requirements. An optimal network selection is proposed in Sehgal and Agarval [10] using distance function to generate an ordered list of available networks. Since it considers the difference between the parameters available and required by the services, it requires frequent handoffs to maintain the connection.

Amali and Ramachandran [11] proposed a modifiedweight-function-based network selection algorithm that considers user preference, network conditions and application profile to evaluate the utility function of the networks. Chamodrakas and Martakos [12] proposed an energy-efficient network selection method, which incorporates the use of utility function to model the diverse QoS elasticity of different applications. Nquyen-Vuong et al [13] considered a multicriteria-utility-function-based network selection method approach that uses sigmoidal function to determine the elementary utility of each attribute. But the weight values are not adapted according to the user preference and application profile.

Wang and Kuo [14] provided various mathematical models and theories such as utility theory, fuzzy logic and game theory in order to achieve the optimization between accuracy and complexity in network selection problems. Khan and Han [15] proposed a VHD scheme to provide a generic connectivity in heterogeneous wireless networks. A simple additive weighting method is used to evaluate the available networks and the Technique for Order of Preference by Similarity to Ideal Solution model is used for network selection, considering the parameters such as delay, data rate, bit error rate and packet loss ratio. However, simple additive weighting method cannot provide guaranteed QoS for mobile users.

Amali et al [16, 17] presented a network selection scheme based on enhanced media-independent handover (EMIH) to facilitate seamless connectivity with guaranteed QoS and minimal handover rate. EMIH framework provides complete information about the link layer and application layer to handover decision module in order to provide optimal network selection to mobile users. In Amali and Ramachandran [18], an intelligent fuzzy logic integrated with fuzzy analytic hierarchy process and principal component analysis is proposed to reduce the computational cost in multiattribute decision-making algorithms for VHD in heterogeneous wireless networks. 
The trade-off between computational cost and consistency of network selection is achieved by reducing the number of unnecessary handovers in comparison with fuzzy-logicbased approaches. In [19], the challenges issued by NGWN due to the diverse characteristics of networks and the shortcoming of traditional wireless communication networks in making connectivity ubiquitous and pervasive are clearly highlighted.

A VHO necessity is estimated in Abdoulaziz et al [20], where travelling time prediction and time threshold calculations are carried out to minimize handover failures and unnecessary handover between cellular networks and WLAN. This method can estimate only the travelling time between entry and exit points on the WLAN cell and does not consider the remaining residence time available to the user inside the WLAN. This issue is considered in our proposed approach to improve the performance of the network selection algorithm. Hussain et al [21] proposed handover necessity estimation (HNE), which estimates the necessity of VHO to WLAN based on angle of arrival, and maintains the probability of handover failure and probability of unnecessary handovers within acceptable limits. In this method, network conditions and user preferences are not taken into account to select the target network in heterogeneous wireless networks. In Amali et al [16, 17], a multicriteria network selection algorithm based on multiplicative weighted utility function is proposed to provide complete solution for seamless connectivity in heterogeneous environment based on network conditions, application QoS, MT battery level and user preferences. However, the impact of velocity on the network selection process is not considered.

After an extensive literature review, it is found that the utility function has been used to select the optimum network in order to satisfy the QoS requirements. Utility function and mobility characteristics (velocity and direction) have been analysed separately till now for the evaluation of networks. The probability of handover failures and unnecessary handovers increases as the multi-interface MT moves through the integration of different access networks [22]. Therefore, MT location information like velocity and direction is mandatory for high-velocity users while formulating network selection algorithm in order to avoid unnecessary handovers. To address this problem, utility function based on multicriteria is combined with RRT estimation to minimize the number of handovers in the proposed approach. From this point of view, our work is more innovative in minimizing the number of handover failures and unnecessary handovers.

\section{Framework of the proposed system}

The challenging feature in heterogeneous wireless networks is to provide prolonged cost-effective services to the mobile users with guaranteed QoS and minimal handover rate. Here, multi-criteria optimization using multiplicative utility function is integrated with RRT estimation to select the optimal network based on the network conditions, MT characteristics, QoS requirements and user preferences. The proposed scheme is implemented with two main streams (i) pedestrian users and (ii) high-velocity users, in order to enjoy the benefits of diverse networks. Average pedestrian speed, i.e., $1.5 \mathrm{~m} / \mathrm{s}$, is used to categorize the users. For pedestrian users $(v<1.5 \mathrm{~m} / \mathrm{s})$, network selection is performed only based on multiplicative utility function considering QoS (delay and data rate), energy consumption and cost. However, for high-velocity users $(v>1.5 \mathrm{~m} / \mathrm{s})$, RRT and ARTT are calculated in order to check for the necessity of handover. If the residence time is greater than ARTT, then a handover is performed to the target network for highvelocity users. If the network with maximum utility function serves the network, MT remains connected with serving network to provide trade-off between service quality and continuity in heterogeneous environment.

The system model of the proposed scheme consists of four modules: 1. handover initiation module, 2. network evaluation module, 3. RRT estimation module and 4. network selection module.

Whenever handover is initiated, multi-interface MT discovers available networks in the coverage area and collects the information about the network conditions (data rate, delay, cost and energy consumption) for the discovered networks, mobility characteristics, QoS profile and user preference. The two main functions of the algorithm are network evaluation using multiplicative weighted utility function and RRT estimation for the integration of UMTS, WiMAX and WLAN networks. These two modules are described in the following sections.

\subsection{Network evaluation using multiplicative weighted utility function}

Utility function determines the ability of the network to satisfy the QoS requirements of a particular service. It can be expressed mathematically as a function of attributes and user preferences. User preference reflects the importance of each metric in the selection process according to the requirements of running application (e.g., voice over IP, video streaming applications) and the type of user. In this work, a service-adaptive multiplicative weighted utility function is proposed, which exploits the S-shaped sigmoid function to model the elasticity of applications and to provide optimization among QoS, energy consumption and cost. The elementary utilities of decision metrics ' $x$ ' such as delay, data rate, energy consumption and cost of service are calculated using sigmoidal function [13] given by Eq. (1) with threshold value $\left(x_{m}\right)$ and lower $\left(x_{l}\right)$ and upper $\left(x_{u}\right)$ limits. For each application, there is a minimum and maximum requirement for each criterion $(x)$. The maximum and minimum values of data rate for voice application are 64 and $32 \mathrm{kbps}$, respectively. 


$$
u(x)= \begin{cases}0, & x \leq x_{l} \\ \frac{\left(\frac{x-x_{l}}{x_{m}-x_{l}}\right)^{\zeta}}{1+\left(\frac{x-x_{l}}{x_{m}-x_{l}}\right)^{\zeta}}, & x_{l} \leq x \leq x_{m} \\ 1-\frac{\left(\frac{x_{\mu}-x}{x_{\mu}-x_{m}}\right)^{\zeta}}{1+\left(\frac{x_{\mu}-x}{x_{\mu}-x_{m}}\right)^{\zeta},} & x_{m} \leq x \leq x_{\mu} \\ 1, & x>x_{\mu}\end{cases}
$$

where $\zeta$ determines the user sensitivity to the variation of network characteristics according to the requirements of applications. It should be high for inelastic real-time application to show higher user sensitivity, but small for elastic non-real-time applications. Elementary utility value of QoS is calculated by combining the utility values of delay and data rate. Elementary utility values for the cost of service and energy consumption are also calculated for each network in order to achieve trade-off among QoS, cost and energy consumption during the network selection process.

The existing additive muticriteria utility function is given by

$$
U(x)=\sum_{i=1}^{n} w_{i} u_{i}\left(x_{i}\right) \quad \text { such that } \sum_{i=1}^{n} w_{i}=1
$$

where ' $i$ ' is the number of criteria considered in the network selection and $w_{i}$ are the user preference weights. From this utility model, it can be shown that

$$
\lim _{u_{i}\left(x_{i}\right) \rightarrow 0} U(x) \neq 0
$$

where $u_{i}\left(x_{i}\right)$ represents the elementary utilities of particular network.

For example, consider two networks 1 and 2; let for network 1, elementary utility of QoS is close to zero but the overall utility is high compared to network 2 by using additive utility function. Then, the additive utility function selects network 1 as the target network, which degrades the performance of the system. This effect is known as null utility effect. To overcome this limitation, interdependence among the criteria is taken into account while formulating the multiplicative weighted utility function. To show how it is reflected in the multiplicative utility function, the following conditions must be satisfied:

$$
\begin{gathered}
\frac{\partial U(x)}{\partial u_{i}} \geq 0 \\
\lim _{u_{i} \rightarrow 0} U(x)=0 \quad \forall i=1, \ldots, n \\
\lim _{u_{1}, \ldots, u_{n} \rightarrow 1} U(x)=1
\end{gathered}
$$

Equation (3) reflects the condition that aggregate utility should increase when the elementary utility $u\left(x_{i}\right)$ increases. The proposed aggregate utility function should satisfy Eq. (4) in order to resolve the null utility effect observed in the additive utility function. Thus, it eliminates the access networks having a zero elementary utility in the decision-making process. Equation (5) says that if all the considered criteria satisfy the user requirements and application profile, then the global utility function $U(x)$ should be high.

The overall utility function is calculated for each network using multiplicative utility function $[16,17]$ given by

$$
U(x)=\prod_{i=1}^{4}\left(u_{i}\left(x_{i}\right)\right)^{w_{i}}
$$

where

$$
\begin{aligned}
U(x)= & \left\{\left[u_{\text {delay }}(x)\right]^{w_{1}} \times\left[u_{\text {datarate }}(x)\right]^{w_{2}}\right\} \\
& \times\left[u_{\text {energy }}(x)\right]^{w_{3}} \times\left[u_{\text {cost }}(x)\right]^{w_{4}}
\end{aligned}
$$

$w_{1}, w_{2}, w_{3}$ and $w_{4}$ are the weight values assigned according to the user preferences and QoS profile. Different users may have different preferences to different parameters such as QoS, energy consumption and cost of service. User preferences can be categorized as QoSsensitive, cost-sensitive and energy-sensitive based on the type of user (commercial and consumer users) and MT battery level. If the user requires more quality-oriented services, quality is given a higher priority than other criterion. After the assessment of networks, the available networks are ranked in descending order based on the value of global utility function.

During network evaluation, upward and downward criteria should be distinguished for each network, because users usually prefer high value for upward criteria (data rate) and low value for downward criteria (delay, cost and energy consumption). Downward criteria follow the principle 'the smaller the better'. This may result in low value of utility and affects the overall utility of each network. For example, if the value of delay offered by the network is low, it is more beneficial, i.e., guarantees better QoS to the running applications. To compensate this effect, effective value of downward criteria is obtained as follows:

$$
u_{\text {delay }}(x)=1-u_{1 \text { delay }}(x)
$$

where $u_{1 \text { delay }}(x)$ is the actual elementary utility of delay obtained from Eq. (1).

Since the cost utility is downward criteria, the effective utility value of cost is calculated as

$$
u_{\text {cost }}(x)=1-u_{1 \operatorname{cost}}(x)
$$

where $u_{1 \text { cost }}(x)$ is the actual utility value of the cost for a particular network. 
The energy utility value should be high for low energy consumption. To achieve this, the effective value should be expressed as follows:

$$
u_{\text {energy }}(x)=1-u_{\text {energy }}(x)
$$

where $u_{\text {1energy }}(x)$ is the actual elementary utility value of the energy consumption (power consumption/bit rate). If $E$ is the actual power consumption of the network, then $x_{l}, x_{m}$ and $x_{u}$ values of energy consumption can be determined as follows: $x_{l}=E / 32 \mathrm{kbps}, x_{m}=E / 50 \mathrm{kbps}$ and $x_{u}=$ $E / 64 \mathrm{kbps}$, where 32, 50 and $64 \mathrm{kbps}[13]$ are, respectively, the $x_{l}, x_{m}$ and $x_{u}$ values of data rate for the voice application.

\subsection{Residual residence time estimation and network selection module}

After evaluating the available networks using multiplicative utility function, the proposed algorithm also computes the RRT for the network, which has the maximum utility function. Even though utility values obtained help in selecting the target network, it may lead to unnecessary handovers, especially when there are a large number of overlapping networks. Hence, there is a need to check for the necessity of handovers. A handover necessity check using residence time estimation is proposed to calculate the remaining time available for a MT in a particular network. Hence it is named as RRT estimation. An illustration for showing the importance of RRT calculation is shown in figure 1. In the following analysis, it is assumed that the coverage of UMTS network is ever-present. It is also assumed that current location of MT is point ' $A$ ' and is currently served by the WiMAX network. At point 'A', it can detect signals from base stations of all the three networks, i.e., UMTS, WiMAX and WLAN. It is found that the related works in the literature $[20,21]$ estimate the residence time between the entry and exit point of the MT at the boundary of the network and perform handovers only if

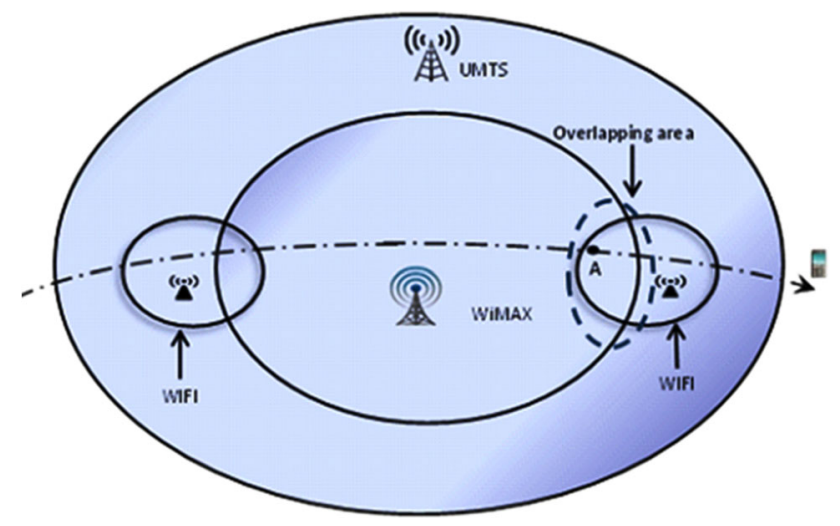

Figure 1. RRT necessity estimation. the estimated residence time is greater than the constant threshold value. Thus, these methods cannot estimate the RRT available to the users along its trajectory, whereas the proposed analytical approach estimates the residence of MT at any point on the trajectory inside the network and performs handover only if the estimated residence time is greater than the adaptive threshold value.

The sequence of operations to be carried out for the implementation of the proposed system is shown in figure 2. For pedestrian users, then the network with maximum utility function in the list is selected as target network whereas for high-velocity users $(v>1.5 \mathrm{~m} / \mathrm{s})$, RRT and ARTT are calculated in order to check for the necessity of handover. If the residence time is greater than ARTT, then handover is performed to the target network for high-velocity users. If the network with maximum utility function is serving the network, MT remains connected with serving network to provide trade-off between service quality and continuity in heterogeneous environment.

3.2a RRT estimation: RRT is calculated particularly if there is an overlapping region of available networks. Let points $\mathrm{C}$ and $\mathrm{E}$ be the current and the exit locations of the MT in the network as shown in figure 3 and a straight line trajectory is assumed for the movement of MT. The points $\mathrm{C}$ and $\mathrm{E}$ are arbitrarily chosen with equal probability. Then the current and exit angles $\theta_{c}$ and $\theta_{e}$ are uniformly distributed in the interval $[0 ., . \pi)$ and the angle between the current location and the exit point is named as residue angle, $\theta=\theta_{c}-\theta_{e}$. The maximum value of $\theta_{c}$ and $\theta_{e}$ is $\pi$ as it corresponds to a trajectory along the diameter of the network.

Since the current location and the exit points are independent of each other, the joint probability distribution function (PDF) is given as

$$
f\left(\Theta_{c}, \Theta_{e}\right)= \begin{cases}\frac{1}{\pi^{2}} & 0 \leq\left(\Theta_{c}, \Theta_{e}\right) \leq \pi \\ 0 & \text { otherwise }\end{cases}
$$

The probability that $\theta \leq \Theta$ which is also the cumulative distribution function of $\theta$, can be derived as follows:

$$
F(\Theta)=P(\theta \leq \Theta)
$$

$P(\theta \leq \Theta)$ is derived using the integral used in Bettstetter et al [23] and $F(\Theta)$ is obtained as

$$
F(\Theta)=\frac{2 \Theta}{\pi}+\frac{\Theta^{2}}{\pi^{2}}
$$

The PDF of $\theta$ can be derived by taking the derivative of $F(\Theta)$ :

$$
f(\Theta)=\frac{2}{\pi}\left[1+\frac{\Theta}{\pi}\right] .
$$

Let figure 3 represent an access network with radius ' $r$ ' and the base station at the centre ' $\mathrm{O}$ '; ' $d$ ' is the distance between the current location of MT and the base station. The distance ' $s$ ' represents the remaining distance to be covered by the MT. 


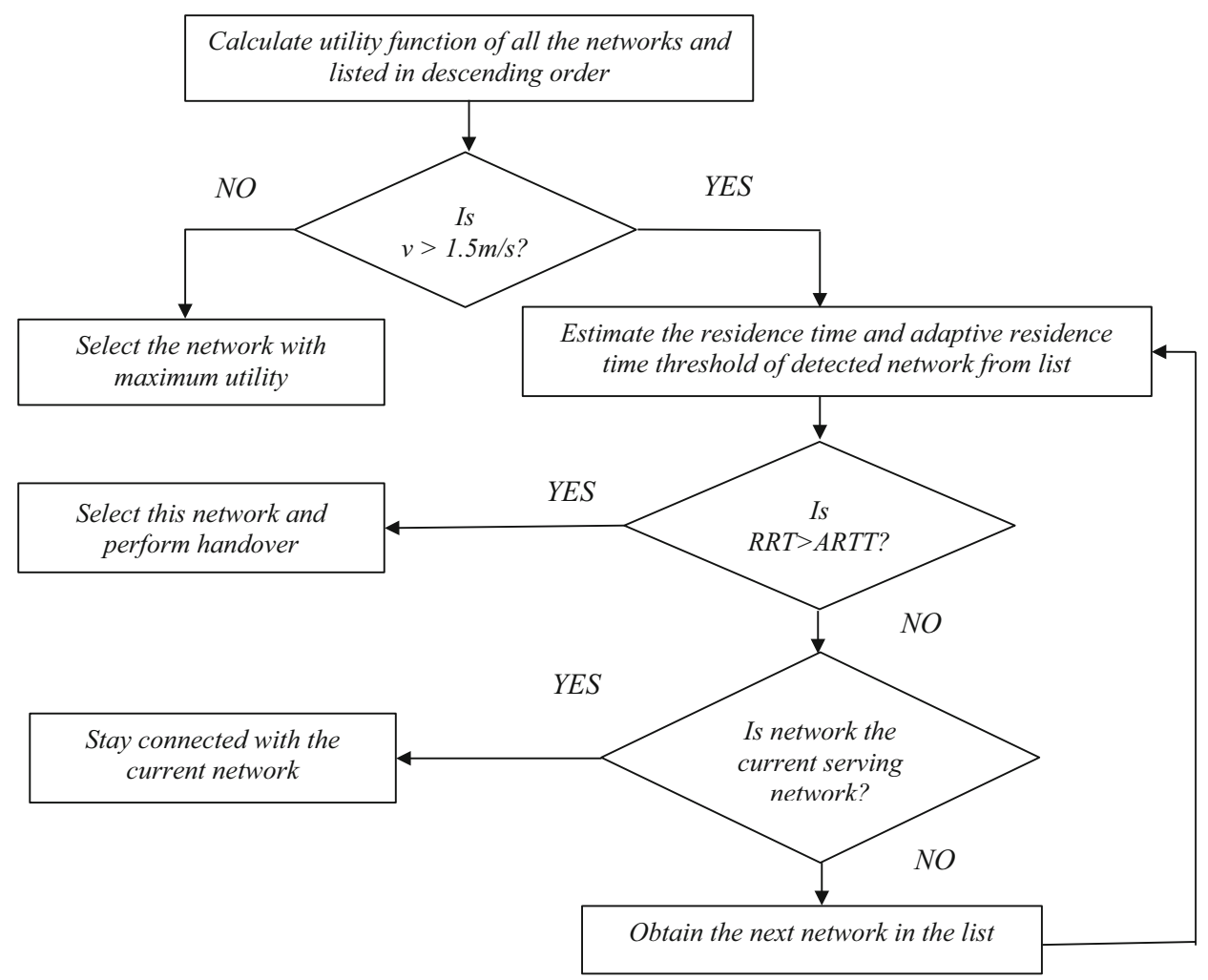

Figure 2. Flowgraph of proposed network selection scheme.

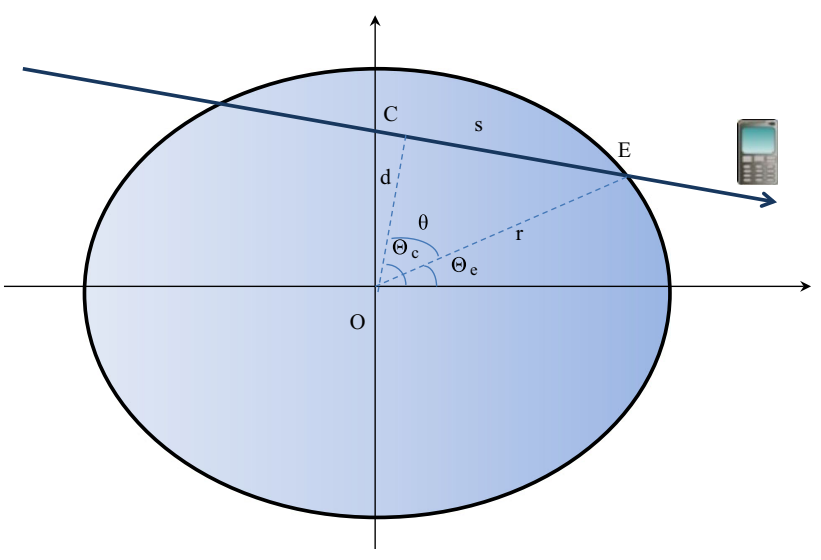

Figure 3. Schematic diagram for RRT calculation.

Using the cosine theorem in $\triangle \mathrm{COE}$

$$
s=\sqrt{\boldsymbol{r}^{2}+\boldsymbol{d}^{2}-2 \boldsymbol{r d} \cos \boldsymbol{\theta}} .
$$

The RRT ' $t$ ' of the MT in the network can be written as

$$
t=\frac{s}{v}
$$

where ' $v$ ' is the velocity of the MT.

Substituting (15) in (16)

$$
t=\frac{\sqrt{r^{2}+d^{2}-2 \boldsymbol{r d} \cos \theta}}{v}=g(\theta) .
$$

Thus, (17) gives the RRT of the MT in a particular access network. To estimate the ARTT, the PDF of ' $\mathbf{t}$ ' should be calculated.

The derivative of $g(\theta), g^{\prime}(\theta)$, is obtained as follows:

$$
g^{\prime}(\theta)=\frac{r d \sin \theta}{v \sqrt{r^{2}+d^{2}-2 r d \cos \theta}}
$$

Using the theorem stated in Kay [24] and Jay Kerns [25]

$$
f(T)=\sum_{i=1}^{\text {number of roots }} \frac{f(\theta)}{\left|g^{\prime}(\theta)\right|} .
$$

There is only a single root for $\theta$ as $\theta$ lies in the interval $[0, \pi)$. Hence, substituting the value of $\theta$ from (17), $f(\theta)$ and $g^{\prime}(\theta)$ is obtained as follows:

$$
f(\theta)=\frac{2}{\pi}\left(1+\frac{\cos ^{-1}\left(\frac{r^{2}+d^{2}-v^{2} T^{2}}{2 r d}\right)}{\pi}\right)
$$

$$
g^{\prime}(\theta)=\frac{r d \sin \left(\cos ^{-1}\left(\frac{r^{2}+d^{2}-v^{2} t^{2}}{2 r d}\right)\right)}{v \sqrt{r^{2}+d^{2}-2 r d \cos \left(\cos ^{-1}\left(\frac{r^{2}+d^{2}-v^{2} t^{2}}{2 r d}\right)\right)}}
$$

$\left|g^{\prime}(\theta)\right|$ can be written as 


$$
\left|g^{\prime}(\theta)\right|=\frac{\sqrt{4 r^{2} d^{2}-\left(r^{2}+d^{2}-v^{2} t^{2}\right)^{2}}}{2 v^{2} t} .
$$

Substituting (20) and (22) in (19), the PDF of RRT is obtained as

$$
f(T)=\frac{2 v^{2} t\left(1+\frac{\cos ^{-1}\left(\frac{r^{2}+d^{2}-v^{2} T^{2}}{2 r d}\right)}{\pi}\right)}{\pi r d \sqrt{1-\frac{\left(r^{2}+d^{2}-v^{2} T^{2}\right)^{2}}{(2 r d)^{2}}}} .
$$

It is inferred that the residence time available for the MT inside the network depends on the network coverage $(r)$, distance $(d)$, residue angle $(\theta)$ and velocity of the MT $(v)$. The proposed analytical model uses the PDF of RRT to obtain the ARTT values for probability of handovers, handover failures and unnecessary handovers.

3.2b ARTT for probability of handover, $\mathrm{P}_{\mathrm{h}}$ : A handover is performed only when the residence time of a network is greater than ARTT $\left(T_{1}\right)$ in order to minimize the probability of handovers. Therefore, the probability of handover can be expressed as follows:

$$
\begin{gathered}
P_{h}= \begin{cases}P\left(T \geq T_{1}\right), & 0 \leq T \leq \frac{2 r}{v} \\
0 & \text { otherwise }\end{cases} \\
P_{h}=\int_{T_{1}}^{T_{\max }} f(T) d t
\end{gathered}
$$

where $T_{\max }$ is the maximum time during which a MT can reside inside the network. Thus

$$
P_{h}=1-\int_{T_{\text {min }}}^{T_{1}} f(T) d t
$$

where $T_{\min }=\frac{2 r}{v_{\max }}$ is the minimum time for which a MT can dwell in a particular access network and $v_{\max }$ is the maximum velocity of the MT.

Substituting (23) in (26), $P_{h}$ can be obtained as follows:

$$
\begin{gathered}
P_{h}=1-\int_{T_{\min }}^{T_{1}} \frac{2 v^{2} t\left(1+\frac{\cos ^{-1}\left(\frac{r^{2}+d^{2}-v^{2} T^{2}}{2 r d}\right)}{\pi}\right)}{\pi r d \sqrt{1-\frac{\left(r^{2}+d^{2}-v^{2} T^{2}\right)^{2}}{(2 r d)^{2}}}} d T \\
P_{h}=1-\left(\frac { 2 } { \pi } \left(\cos ^{-1}\left(\frac{r^{2}+d^{2}-v^{2} T_{1}^{2}}{2 r d}\right)\right.\right. \\
\left.-\cos ^{-1}\left(\frac{r^{2}+d^{2}-v^{2} T_{\min }^{2}}{2 r d}\right)\right) \\
+\frac{1}{\pi^{2}}\left(\left(\cos ^{-1}\left(\frac{r^{2}+d^{2}-v^{2} T_{1}^{2}}{2 r d}\right)\right)^{2}\right. \\
\left.\left.-\left(\cos ^{-1}\left(\frac{r^{2}+d^{2}-v^{2} T_{\min }^{2}}{2 r d}\right)\right)^{2}\right)\right) .
\end{gathered}
$$

$\operatorname{ARTT}\left(T_{1}\right)$ is obtained as

$T_{1}=\frac{\sqrt{r^{2}+d^{2}-2 r d \cos \left(-\pi \pm \sqrt{k_{h}^{2}+2 \pi k_{h}+\left(2-P_{h}\right) \pi^{2}}\right)}}{v}$

where $k_{h}=\cos ^{-1}\left(\frac{r^{2}+d^{2}-v^{2} T_{\text {min }}^{2}}{2 r d}\right)$.

Using (29), ARTT $\left(T_{1}\right)$ is calculated as a function of velocity $(v)$, minimum residence time of MT in a network $\left(T_{\min }\right)$ and given value of $P_{h}$. Thus, the use of ARTT $\left(T_{1}\right)$ as a decision metric minimizes the probability of handovers for high-velocity users.

3.2c ARTT for probability of handover failure, $\mathrm{P}_{\mathrm{f}}$ : A handover failure occurs when the handover latency in a network, $\tau_{i n}$, is greater than the RRT. A handover is initiated only if the RRT of that particular network is greater than the ARTT $\left(T_{2}\right)$. The threshold is named as adaptive since it changes with the velocity of the MT and handover latency. Considering these conditions, the probability of handover failure can be written as

$$
\begin{gathered}
P_{f}= \begin{cases}P\left(T_{2} \leq T \leq \tau_{\text {in }}\right), & 0 \leq T \leq \frac{2 r}{v} \\
0 & \text { otherwise }\end{cases} \\
P\left(T_{2} \leq T \leq \tau_{\text {in }}\right)=\int_{T_{2}}^{\tau_{i n}} f(T) d T \\
P_{f}=\frac{2}{\pi}\left(\cos ^{-1}\left(\frac{r^{2}+d^{2}-v^{2} \tau_{i n}^{2}}{2 r d}\right)\right. \\
\left.-\cos ^{-1}\left(\frac{r^{2}+d^{2}-v^{2} T_{2}^{2}}{2 r d}\right)\right) \\
+\frac{1}{\pi^{2}}\left(\left(\cos ^{-1}\left(\frac{r^{2}+d^{2}-v^{2} \tau_{\text {in }}^{2}}{2 r d}\right)\right)^{2}\right. \\
\left.-\left(\cos ^{-1}\left(\frac{r^{2}+d^{2}-v^{2} T_{2}^{2}}{2 r d}\right)\right)^{2}\right) .
\end{gathered}
$$

ARTT $\left(T_{2}\right)$ can be written as

$T_{2}=\frac{\sqrt{r^{2}+d^{2}-2 r d \cos \left(-\pi \pm \sqrt{k_{f}^{2}+2 \pi k_{f}+\left(1-P_{f}\right) \pi^{2}}\right)}}{v}$

where $k_{f}=\cos ^{-1}\left(\frac{r^{2}+d^{2}-v^{2} \tau_{i n}^{2}}{2 r d}\right)$.

Thus, ARTT $\left(T_{2}\right)$ is obtained as a function of velocity $(v)$ and handover latency in the network $\left(\tau_{i n}\right)$ to ensure that estimated RRT is greater than $\tau_{\text {in }}$ in order to reduce the probability of handover failures.

$3.2 \mathrm{~d}$ ARTT for probability of unnecessary handover, $\mathrm{P}_{\mathrm{u}}$ : An unnecessary handover occurs when the RRT is less than the total handover latency in and out of the network, 
$\tau_{\text {tot. }}$ Such a situation occurs whenever the travelling time of MT inside a network is very small. Hence, handover to such a network will be futile. To keep the number of unnecessary handovers within limits, an ARTT $\left(T_{3}\right)$ is defined and handover is initiated only if the RRT is greater than ARTT $\left(T_{3}\right)$. The probability of unnecessary handover can be expressed as

$$
P_{u}= \begin{cases}P\left(T_{3} \leq T \leq \tau_{\text {tot }}\right), & 0 \leq T \leq \frac{2 r}{v} \\ 0 & \text { otherwise }\end{cases}
$$

where $\tau_{\text {tot }}=\tau_{\text {in }}+\tau_{\text {out }}$.

$$
\begin{gathered}
P\left(T_{3} \leq T \leq \tau_{\text {tot }}\right)=\int_{T_{3}}^{\tau_{\text {tot }}} f(T) d T \\
\therefore P_{u}=\int_{T_{3}}^{\tau_{\text {tot }}} \frac{2 v^{2} t\left(1+\frac{\cos ^{-1}\left(\frac{r^{2}+d^{2}-v^{2} T^{2}}{2 r d}\right)}{\pi}\right)}{\pi r d \sqrt{1-\frac{\left(r^{2}+d^{2}-v^{2} T^{2}\right)^{2}}{(2 r d)^{2}}} d T} \\
P_{u}=\frac{2}{\pi}\left(\cos ^{-1}\left(\frac{r^{2}+d^{2}-v^{2} \tau_{t o t}^{2}}{2 r d}\right)\right. \\
\left.-\cos ^{-1}\left(\frac{r^{2}+d^{2}-v^{2} T_{3}^{2}}{2 r d}\right)\right) \\
+\frac{1}{\pi^{2}}\left(\left(\cos ^{-1}\left(\frac{r^{2}+d^{2}-v^{2} \tau_{t o t}^{2}}{2 r d}\right)\right)^{2}\right. \\
\left.-\left(\cos ^{-1}\left(\frac{r^{2}+d^{2}-v^{2} T_{3}^{2}}{2 r d}\right)\right)^{2}\right) .
\end{gathered}
$$

ARTT $\left(T_{3}\right)$ is derived from (37) as

$T_{3}=\frac{\sqrt{r^{2}+d^{2}-2 r d \cos \left(-\pi \pm \sqrt{k_{u}^{2}+2 \pi k_{u}+\left(1-P_{u}\right) \pi^{2}}\right)}}{v}$

where $k_{u}=\cos ^{-1}\left(\frac{r^{2}+d^{2}-v^{2} \tau_{t o t}^{2}}{2 r d}\right)$.

ARTT $\left(T_{3}\right)$ is computed for a particular value of probability of unnecessary handovers $\left(P_{u}\right)$ and total handover latency in and out of the network $\left(\tau_{t o t}\right)$ to minimize the ping-pong effects.

\section{Performance evaluation}

In this section, the performance of the proposed integrated scheme is studied analytically considering the integration of UMTS, WiMAX and WLAN networks as a heterogeneous environment as shown in figure 1. Each module of the proposed network selection scheme is evaluated separately in order to show its impact on the network selection decision.

\subsection{Network evaluation module}

The performance analysis of additive and multiplicative utility functions is performed in Amali et al [16, 17] in order to show the efficiency of multiplicative utility over additive utility in network selection strategies. Elementary utility value is computed for each attribute using sigmoidal function using Eq. (1). Tables 1 and 2 show the weight adaption for voice application and video streaming according to the type of users while maintaining the minimum QoS requirements of service. For voice application, higher weight is given for delay whereas for video streaming application, higher weight is given for data rate. However, the weight values are assigned in the utility calculation to satisfy the minimum QoS requirements of service irrespective of the type of user. Thus, the proposed scheme provides optimization among quality, cost and energy during the network selection process. The network parameters are given in table 3 [13]. The proposed scheme considers the minimum and maximum values required to maintain the QoS requirements of applications as defined by 3GPP TS-23.107 specifications [26] as shown in table 4.

Figure 4 shows how the multiplicative utility function selects the target network for pedestrian users according to the QoS requirements and user preference. Table 5 shows the multiplicative utility values obtained for QoS sensitive users for various applications. For QoS-sensitive users, it is observed that UMTS has a higher utility value for voice

Table 1. Weight values for voice application.

\begin{tabular}{lllll}
\hline User preferences & $w_{1}$ & $w_{2}$ & $w_{3}$ & $w_{4}$ \\
\hline QoS & 0.4 & 0.2 & 0.2 & 0.2 \\
Cost & 0.3 & 0.1 & 0.1 & 0.5 \\
Energy & 0.3 & 0.1 & 0.5 & 0.1 \\
\hline
\end{tabular}

Table 2. Weight values for video streaming.

\begin{tabular}{lllll}
\hline User preferences & $w_{1}$ & $w_{2}$ & $w_{3}$ & $w_{4}$ \\
\hline QoS & 0.2 & 0.4 & 0.2 & 0.2 \\
Cost & 0.1 & 0.3 & 0.2 & 0.4 \\
Energy & 0.1 & 0.3 & 0.4 & 0.2 \\
\hline
\end{tabular}

Table 3. Network parameters.

\begin{tabular}{lccc}
\hline Criterion & UMTS & WiMAX & WLAN \\
\hline Cost (units/kbps) & $20-60$ & $1-50$ & $1-40$ \\
Energy in active state (J) & 1.2 & 3.5 & 4.5 \\
Data rate (Mbps) & 1 & 10 & 5 \\
Delay (ms) & 10 & 50 & 130 \\
\hline
\end{tabular}


Table 4. Application requirements.

\begin{tabular}{lcccc}
\hline & $\begin{array}{c}\text { D Data rate } \\
\left(x_{l}\right)(\mathrm{kbps})\end{array}$ & $\begin{array}{c}\text { Data rate } \\
\left(x_{u}\right)(\mathrm{kbps})\end{array}$ & $\begin{array}{c}\text { Delay } \\
\left(x_{l}\right) \\
(\mathrm{ms})\end{array}$ & $\begin{array}{c}\text { Delay } \\
\left(x_{u}\right) \\
(\mathrm{ms})\end{array}$ \\
\hline $\begin{array}{l}\text { Voice } \\
\text { Video } \\
\quad \begin{array}{c}\text { conferencing } \\
\text { Video } \\
\text { streaming }\end{array}\end{array}$ & 512 & 5000 & 75 & 150 \\
$\begin{array}{l}\text { Web browsing } \\
\text { Weation }\end{array}$ & 128 & 1000 & 2000 & 4000 \\
\hline
\end{tabular}

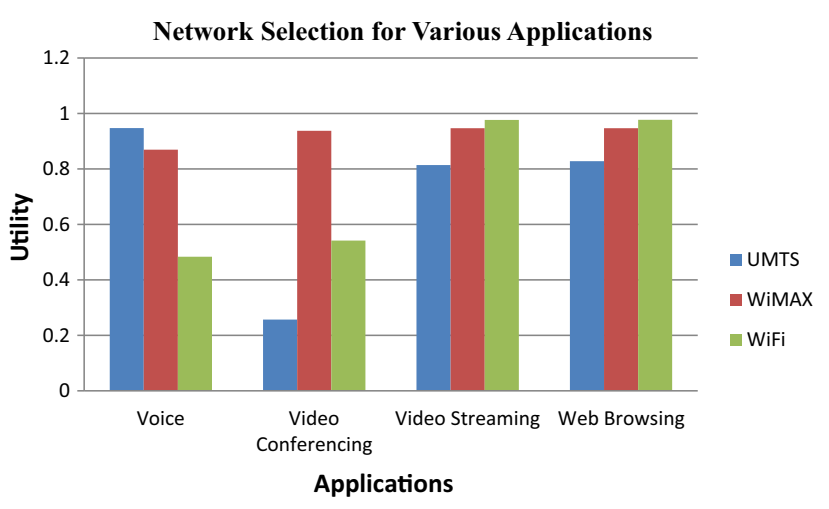

Figure 4. Network selection for pedestrian users.

application considering the delay requirements. Likewise, for the video conferencing application, both UMTS and WiMAX satisfy the delay requirements of the application. However, when the data rate constraints are considered, WiMAX is more desirable than UMTS. As the interdependence between the different criteria is considered in computing multiplicative aggregate utility function, it selects WiMAX as the access network. For both video streaming and web browsing applications, all the three networks satisfy both delay and data rate specifications. Therefore, cost plays an important role in differentiating the available networks. Thus, WLAN is selected for non-realtime applications.

\subsection{RRT estimation module}

To validate the RRT module, the relationship of RRT with the velocity of the MT and the residue angle $\theta$ is demonstrated and the performance of proposed method is compared to the method followed in Hussain et al [21] using Monte-Carlo simulations. To show the importance of ARTT, the adaptive threshold scheme is compared to the constant-threshold scheme. ARTTs $T_{1}, T_{2}$ and $T_{3}$ are computed for a given value of probabilities $P_{h}, P_{f}$ and $P_{u}$, respectively. In the simulation, the angles $\theta_{c}$ and $\theta_{e}$ are generated randomly between 0 and $\pi$ and the residue angle
Table 5. Utility values for QoS sensitive users.

\begin{tabular}{lccc}
\hline & \multicolumn{3}{c}{ Utility values } \\
\cline { 2 - 4 } Application & UMTS & WiMAX & WLAN \\
\hline Voice & 0.8943 & 0.8706 & 0.3689 \\
Video conferencing & 0.2638 & 0.8614 & 0.4364 \\
Video streaming & 0.8364 & 0.8701 & 0.9245 \\
Web browsing & 0.8505 & 0.8702 & 0.9250 \\
\hline
\end{tabular}

$\theta$ is estimated as the difference between $\theta_{c}$ and $\theta_{e}$. The RRT is calculated using (17).

4.2a Relationship among $R R T$, velocity of $M T$ ' $v$ ' and residue angle ' $\theta$ ': Figure 5 shows the variation of residence time with changes in velocity $v$ and residue angle $\theta$. For a fixed velocity of $v=10 \mathrm{~m} / \mathrm{s}$, it can be observed that as residue angle $\theta$ increases the residence time also increases. For $\theta=180^{\circ}$, the MT trajectory will be through the diameter of the network and hence should have maximum residence time. Likewise, when the velocity of the MT increases, the time required to cross a network decreases. It can be noticed that residence time decreases with increase in the velocity of MT. Thus, the adaptive threshold values $T_{1}, T_{2}$ and $T_{3}$ should be determined as a function of velocity, residue angle, residence time and designed value of performance metrics in order to keep the probability of handovers, handover failures and unnecessary handovers within the limits.

4.2b Probability of handovers, $P_{\mathrm{h}}$ : In figure 6 , the variation of probability of handovers with velocity is analysed for the proposed scheme with ARTT as well as constantthreshold scheme and HNE method [21]. For comparison, a similar equation has been derived for the HNE method using (23). To emphasize the importance of using ARTT, RRT estimation with and without ARTT is also compared. For an adaptive threshold scheme, ARTT $\left(T_{1}\right)$ changes adaptively with increase of MT velocity as in (29). Therefore, the number of handovers decreases with a rise in the velocity of MT and thus the probability of handovers. The results demonstrate that our proposed scheme outperforms against the existing methods and reduces the number of handovers by $25 \%$ and $40 \%$ for the velocity of $40 \mathrm{~m} / \mathrm{s}$ when compared with constantthreshold and HNE methods, respectively. In HNE, RRT estimation is performed between the entry and exit points of the MT at the boundary of the network. However, context aware utility calculation was not considered in the network selection process. Thus, the probability of handover is high in HNE method compared with the proposed method with adaptive threshold.

4.2c Probability of handover failure, $P_{\mathrm{f}}$ : The ARTT $\left(T_{2}\right)$ for probability of handover failure is determined using (33) by assigning $P_{f}$ equal to 0.02 and $\tau_{\text {in }}$ as the worst-case handover latency of $1 \mathrm{~s}$ [21]. A handover is attempted 


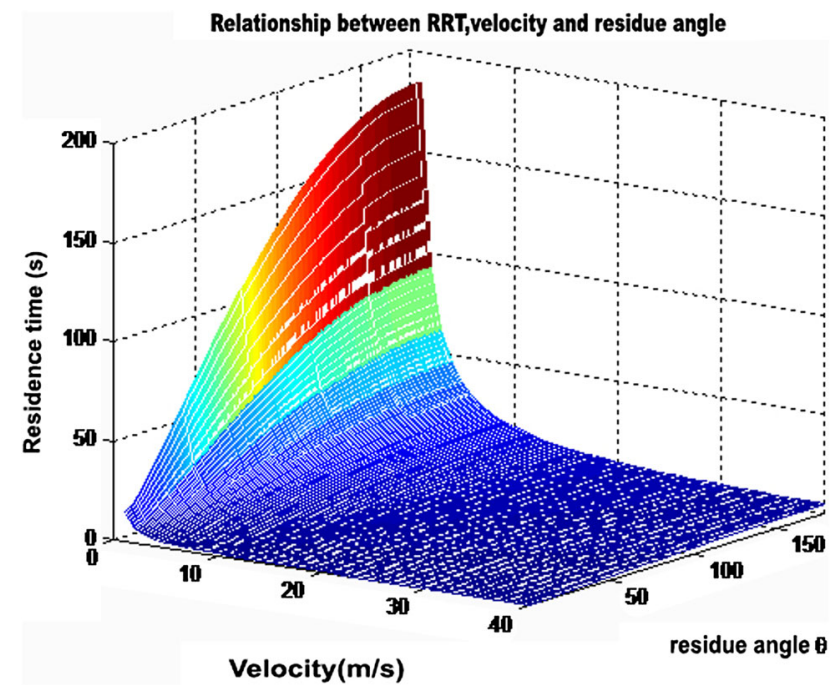

Figure 5. Relationship among RRT, velocity and residue angle.

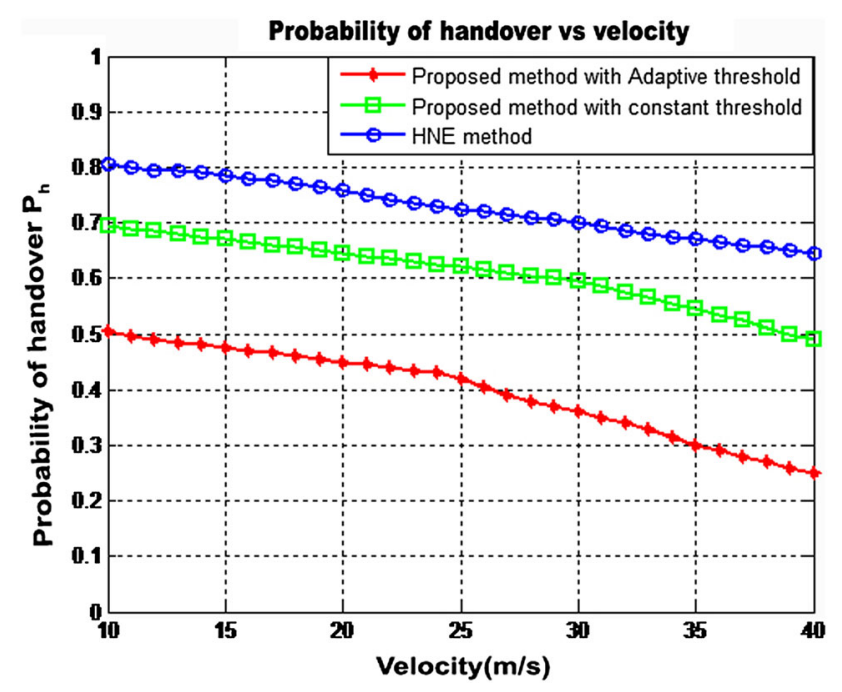

Figure 6. Probability of handovers vs velocity of MT.

whenever the RRT is greater than ARTT $\left(T_{2}\right)$ and it becomes successful whenever RRT is greater than the handover latency to enter into the network. Figure 7 shows the variation of probability of handover failure with velocity of MT. It is clear that the proposed RRT scheme achieves the designed $P_{f}$ of 0.02 compared to the HNE scheme [21]. Probability of handover failures can be obtained as the ratio of number of failed handovers to the number of handover attempts. The experiment is carried out for a large number of times until a clear pattern is obtained using Monte-Carlo simulation.

The proposed method reduces the probability of handover failures by $16 \%$ against the constant-threshold scheme. Hence constant threshold value cannot be used as a decision metric to initiate handover for high-velocity users.

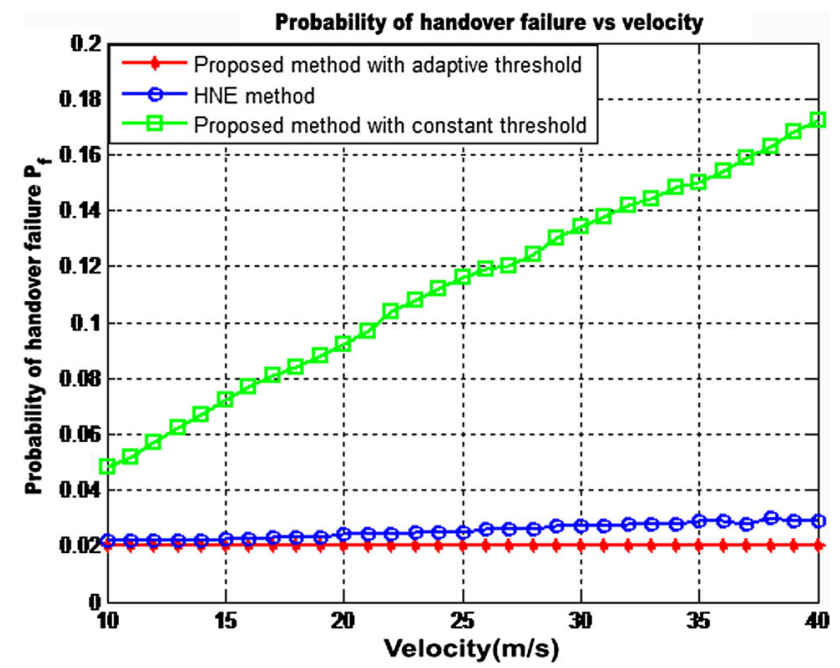

Figure 7. Probability of handover failure vs velocity of MT.

The HNE method considers only mobility context to perform handover decision for the integration of UMTS and Wi-Fi networks. However, service and user context were not considered in the decision-making process. Thus, the amount of deviation from the designed value increases when the velocity of MT increases as shown in figure 7 .

4.2d Probability of unnecessary handovers, $P_{\mathrm{u}}$ : An unnecessary handover occurs when the residence time of MT inside a network is less than total handover latency $\tau_{\text {tot }}$. ARTT $\left(T_{3}\right)$ is determined by assigning values $P_{u}$ as 0.02 and $\tau_{\text {tot }}$ as $2 \mathrm{~s}$ in (38). The results obtained through simulation are shown in figure 8. It is observed that the probability of unnecessary handovers in the proposed adaptive threshold scheme maintains the designed value of 0.02 and is slightly less than that by the HNE method [21] but $8 \%$ less than that by the constant-threshold scheme. The HNE method can only minimize the probability of handover failures and unnecessary handovers. On the other hand, the proposed adaptive threshold scheme can reduce the probability of handovers by $40 \%$ and also keep the probability of handover failures and unnecessary handovers to the designed value of $P_{f}=P_{u}=0.02$.

\subsection{Network selection module}

This section describes the network selection for various applications specifically for QoS-sensitive users. Figure 9 shows the network selection for both pedestrian and highvelocity users. The network with maximum utility value is selected as a target network for pedestrian users. The utility calculation is performed considering the QoS requirements of application, network conditions and user preference as QoS-sensitive. However, the network that satisfies both utility and the residence time constraints is selected as a target network for high-velocity users. For both $v=1.5$ 


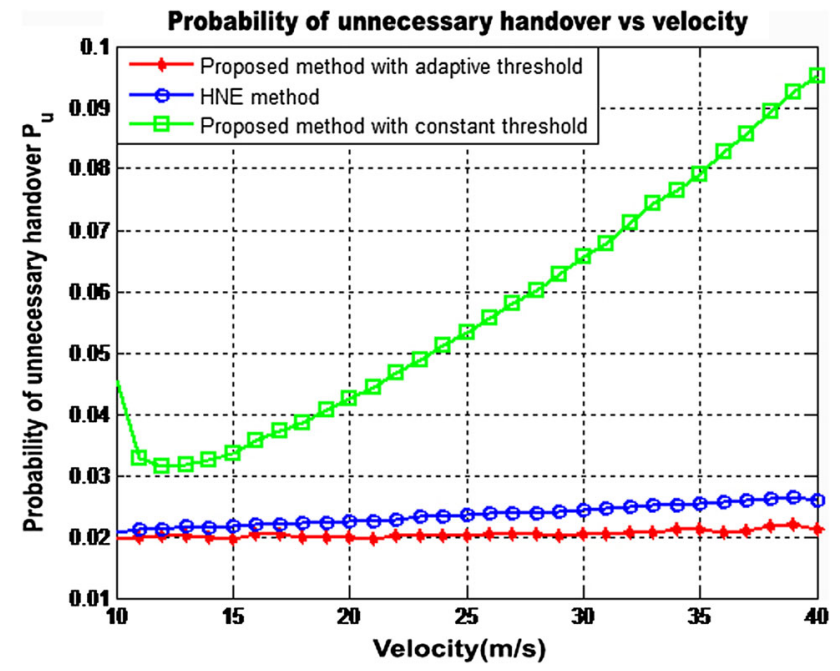

Figure 8. Probability of unnecessary handovers vs velocity of MT.

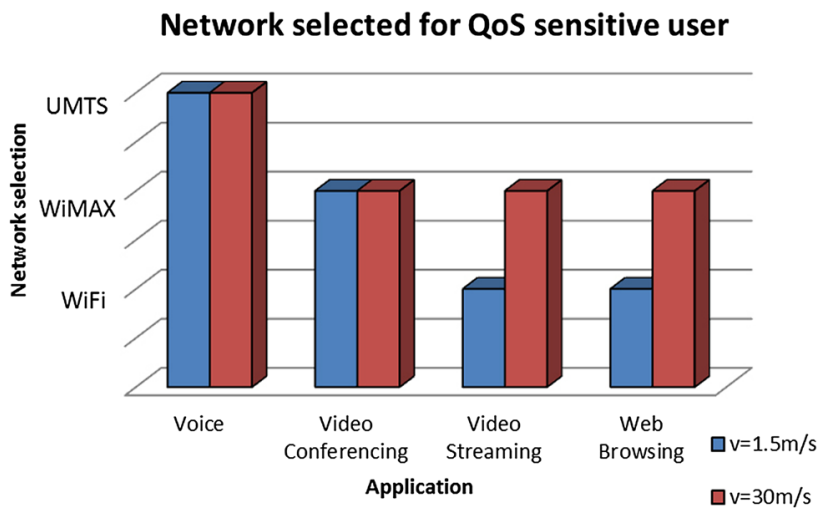

Figure 9. Network selection for both pedestrian and high-velocity users.

$\mathrm{m} / \mathrm{s}$ (pedestrian users) and $v=30 \mathrm{~m} / \mathrm{s}$ (high-velocity users), the MT stays connected with the UMTS and WiMAX for voice and video conferencing applications, respectively, as it satisfies both utility and the residence time conditions. However, for non-real time applications, WLAN is selected as the target network for pedestrian users. However, the algorithm prefers WiMAX as the target network for high-velocity users as it satisfies both utility and the residence time constraints.

\section{Conclusion}

This paper proposes a multicriteria network selection approach based on multiplicative weighted utility function and RRT estimation to provide VHO for interworking among UMTS, WiMAX and WLAN networks. Multiplicative utility function is calculated taking into account network conditions, QoS, user preferences and energy consumption to select the best network in heterogeneous environment. In order to reduce the number of handover failures and unnecessary handovers, a novel RRT estimation method is proposed to estimate the RRT available for a MT at any point inside an access network.

Through Monte-Carlo simulations, it is proved that the proposed adaptive threshold scheme outperforms against constant-threshold scheme by reducing the probability of handovers, handover failures and unnecessary handovers by $25 \%, 16 \%$ and $8 \%$, respectively, for the velocity of $40 \mathrm{~m} / \mathrm{s}$. It is also compared with the performance of HNE method to show that the proposed network selection based on multiplicative utility function and RRT estimation reduces the probability of handovers by $40 \%$ and keeps the probability of handover failures and unnecessary handovers to the designed value of $P_{f}=P_{u}=0.02$. Thus, the proposed adaptive threshold scheme keeps the probability of handovers, handover failures and unnecessary handovers within the acceptable limits. In this work, technology-based pricing scheme is considered to provide solutions for the challenges behind the integration of QoS and mobility support. For further research, the pricing models used by different network operators will be investigated due to the coexistence of multiple service providers and technologies.

\section{References}

[1] Gustaffson E and Jonsson A 2003 Always best connected. IEEE Wirel. Commun. 10(1): 49-55

[2] Nguyen-Vuong Q T, Agoulmine N and Ghamri-Doudane Y 2007 Terminal-controlled mobility management in heterogeneous wireless networks. IEEE Commun. Mag. 45(4): 122-129

[3] Ali T and Saquib M 2011 Performance evaluation of WLAN-cellular media access for mobile voice users under random mobility models. IEEE Trans. Wirel. Commun. 10(10): 3241-3254

[4] Mehbodniya A, Kaleem F, Yen K K and Adachi F 2013 A novel wireless network access selection scheme for heterogeneous multimedia traffic. In: Proceedings of the IEEE Consumer Communication and Networking Conference, Las Vegas, USA, pp. 485-489

[5] Mehbodniya A, Kaleem F, Yen K K and Adachi F 2012 Application of FVIKOR method for prioritization of wireless networks with multiple attributes. In: Proceedings of the 23rd IEEE International Symposium on Personal Indoor and Mobile Radio Communications, Sydney, pp. 915-920

[6] He Q, Chen G and Zhang L 2010 A vertical handoff decision algorithm based on fuzzy control in WiMAX and TD-SCDMA heterogeneous wireless networks. In: Proceedings of the 6th International Conference on Wireless Communications Networking and Mobile Computing, Chengdu, China, pp. 1-4

[7] Singhrova A and Prakash N 2012 Vertical handoff decision algorithm for improved quality of service in heterogeneous wireless networks. IET Commun. 6(2): 211-223

[8] Kaleem F, Mehbodniya A, Yen K K and Adachi F 2013 A fuzzy preprocessing module for optimizing the access 
network selection in wireless networks. J. Adv. Fuzzy Syst. Article ID 232198:1-9

[9] Amali C, Bibin Mathew and Ramachandran B 2013 Intelligent network selection using fuzzy logic for $4 \mathrm{G}$ wireless networks. Int. J. Electron. Commun. Eng. Technol. 4(2): 451-461

[10] Sehgal A and Agarval R 2010 QoS based network selection scheme for $4 \mathrm{G}$ systems. IEEE Trans. Consum. Electron. 56(2): 560-565

[11] Amali C and Ramachandran B 2012 Modified weight function based network selection algorithm for $4 \mathrm{G}$ wireless networks. In: Proceedings of the International Conference on Advances in Computing, Communication and Informatics, Chennai, India, pp. 292-299

[12] Chamodrakas I and Martakos D 2012 A utility-based fuzzy TOPSIS method for energy efficient network selection in heterogeneous wireless networks. J. Appl. Soft Comput. 12(7): 1929-1938

[13] Nquyen-Vuong Q T, Agoulmine N, Cherkaoui E H and Toni L 2013 Multicriteria optimization of access selection to improve the quality of experience in heterogeneous wireless access networks. IEEE Trans. Veh. Technol. 62(4): $1785-1800$

[14] Wang L and Kuo G S 2013 Mathematical modeling for network selection in heterogeneous wireless networks - a tutorial. IEEE Commun. Surv. Tut. 15(1): 271-292

[15] Khan M and Han K 2015 A vertical handover management scheme based on decision modelling in heterogeneous wireless networks. IETE Tech. Rev. 32(6): 402-412

[16] Amali C, Jayaprakash D and Ramachandran B 2014 Enhanced media independent network selection for heterogeneous wireless networks. IETE Tech. Rev. 31(5): 392-401
[17] Amali C, Jayaprakash D and Ramachandran B 2014 Optimized network selection using aggregate utility function in heterogeneous wireless networks. Int. Rev. Comput. Softw. 9(7): 1293-1301

[18] Amali C and Ramachandran B 2016 Complexity-consistency trade-off in multi-attribute decision making for vertical handover in heterogeneous wireless networks. IET Netw. 5(1): 13-21

[19] Ahmed A, Boulahia L M and Garti D 2014 Enabling vertical handover decisions in heterogeneous wireless networks: a state-of-the-art and a classification. IEEE Commun. Surv. Tut. 16(2): 776-811

[20] Abdoulaziz I H, Renfa L and Fanzi Z 2012 Handover necessity estimation for $4 \mathrm{G}$ heterogeneous networks. Int. J. Inf. Sci. Tech. 2(1): 1-13

[21] Hussain R, Malik S A, Adrar S, Riaz R A, Ahmed H and Khan S A 2013 Vertical handover necessity estimation based on a new dwell time prediction model for minimizing unnecessary handovers to a WLAN cell. J. Wirel. Pers. Commun. 71(2): 1217-1230

[22] Wang H, Laurenson D I and Hillston J 2013 A general performance evaluation framework for network selection strategies in 3G-WLAN interworking networks. IEEE Trans. Mob. Comput. 12(5): 868-884

[23] Bettstetter C, Hartenstein H and P'erez-Costa X 2004 Stochastic properties of the random waypoint mobility model. J. Wirel. Netw. 10(5): 555-567

[24] Kay S M 2006 Intuitive probability and random process using MATLAB. Springer Verlag, US

[25] Jay Kerns G 2011 Introduction to probability and statistics using $R, 1$ st ed. ISBN: 978-0-557-24979-4

[26] Rinne J 1999 3GPP specification details. TS 23.107 